\title{
SEESAW NYSTAGMUS*
}

\author{
BY \\ O. A. JENSEN \\ Copenhagen County Hospital, Eye Department, Hellerup, Denmark
}

SEESAW nystagmus is a rare disjunctive form of vertical nystagmus in which the eyes perform opposed vertical movements, i.e. while one eye moves up the other moves down. Larsen (1947) reported a case and the autopsy findings, together with a review of previous cases.

We have recently seen two additional cases, and as the condition is extremely rare, it seems.justifiable to report them.

\section{Case Reports}

Case 1, a works foreman aged 55, had always been in good health apart from an operation for left-sided otitis media in 1931.

One evening, when sitting in his home after supper, he suddenly developed, without any preceding symptoms, a feeling of numbness in the left half of the tongue, spreading to the left side of the face, uncharacteristic giddiness, diplopia, and a desire to defaecate. He did not lose consciousness, nor experience headache or convulsions.

When admitted to the medical department the same evening, the patient was torpid, lying with his eyes closed, but gave adequate answers when addressed.

Examination.-Blood pressure $160 / 90 \mathrm{~mm}$. $\mathrm{Hg}$; pulse rate 95 ; rectal temperature $36 \cdot 6^{\circ} \mathrm{C}$. $\left(98^{\circ} \mathrm{F}\right.$.). No neck rigidity, but deviation of the tongue to the left; hypaesthesia of the left side of the face and left half of the tongue; exaggerated reflexes on the right lower limb. The plantar response was doubtful. There were no sensory changes on the trunk or limbs. Lumbar puncture showed a pressure of $140 \mathrm{~mm}$. $\mathrm{Hg}$ with elevation upon Queckenstedt's test. The cell count was $29 / 3$ red cells, $4 / 3$ polynuclears, and 5/3 mononuclears. The protein values were normal.

Ophthalmic Examination.-On the following day the visual acuity was 6/18 with $+1 \mathrm{D} \mathrm{sph}$. in both eyes. The position of the eyes was most of the time highly divergent. This applied particularly to the left eye, in which the angle of deviation varied from 15-60 degrees. At the same time, a pronounced, irregular nystagmus was observed. It was predominantly of the vertical type and was more severe on the left side. There were frequent opposed movements of the two eyes, the rare so-called seesaw nystagmus, and a few horizontal, rotatory movements. Paresis of the left medial rectus was observed, but diplopia tests by means of coloured glasses were not practicable owing to the irregular ocular movements. The pupils were normal. Left-sided corneal hypaesthesia was present. Ophthalmoscopy revealed no abnormality, especially no sign of choked discs. The visual fields could not be determined because the patient was unable to fix the gaze.

Otological Examination.-There was slight hearing loss of the perceptive type on the right, and severe impairment, mainly of the conduction type, on the left. Vestibular reaction was weakened on the left. The aural abnormality was thought to be inactive and unrelated to the present disease.

Laboratory Examination.-The ordinary blood and sero-reactions, and $x$ rays of the skull showed no abnormality.

The neurologist concluded that this must be a case of thrombosis or some other occlusive disease either of the left posterior inferior cerebellar artery or of some other artery within the area supplied by the basilar artery. 
Ophthalmic examination 2 days later showed the visual acuity in both eyes to be 6/6 with $+1 \mathrm{D}$ sph. The nystagmus had disappeared and the paresis of the rectus had subsided. The corneal sensitivity, the ophthalmoscopic findings, and the visual fields were normal, and the patient was now feeling well. All the other neurological manifestations had disappeared, and the patient was discharged after having spent 3 weeks in hospital. The anticoagulant therapy which had been instituted immediately after admission to hospital was continued.

Case 2, a 66-year-old man, had been in good health apart from uncomplicated diphtheria during childhood and numerous uncomplicated gonococcal infections. He denied syphilis. For many years, he had had an excessive daily consumption of strong alcoholic liquors.

On the day before admission, when the patient woke up, he felt giddy and saw double. His own doctor called an ophthalmologist as well as a neurologist who found paresis of the left medial rectus and typical almost purely seesaw nystagmus with only a slight rotatory component. There were other neurological signs apart from tremor of the hands. The patient was hyperactive and restless.

When admitted to the Eye Department, he was quieter and better orientated, but showed unmistakable signs of inebriation.

Ophthalmological Examination.-Vision in the right eye was $6 / 18$ with $+1.5 \mathrm{D}$ sph., and in the left eye $6 / 9$ with $+1.5 \mathrm{D}$ sph. The right eye was in a position of a somewhat varying divergence with perhaps slightly restricted adduction and some tendency to sursumvergence. A distinct paresis of the left medial rectus muscle was present. On changing the direction of the gaze, the ocular movements were fluttering and at times nystagmoid with opposed vertical movements. Occasionally, the upward as well as the downward movements appeared to be inhibited. The pupils reacted to light. Apart from slight cataractous changes, slit-lamp and ophthalmoscopic examinations showed no abnormalities. The visual fields for white and red objects appeared to be normal, but co-operation was poor.

Neurological Examination.-There was only tremor of the hands and a weak extensor plantar response on the right side. Shortly after admission the patient insisted upon being discharged and he has since been lost sight of.

\section{Discussion}

Seesaw nystagmus is very rare (Duke-Elder, 1949; Walsh, 1947). Three cases have been reported, one of them examined post mortem. Cords (1930) observed seesaw movements in cases of miners' nystagmus.

The reported cases do not give much aid in elucidating the pathogenesis.

Maddox (1914) published the first case which was associated with somewhat weak knee jerks and bitemporal hemianopia. The upward movement of the right eye and the downward movement of the left eye were accompanied by a conjugate torsion of the eyeball to the left, and the downward movement of the right eye and the upward movement of the left were accompanied by a parallel conjugate torsion to the right.

In the patient reported by Rucker (1946), the movements of the right eye were almost entirely vertical, while the left eye also showed intorsion with the upward phase and extorsion with the downward phase. The pupils did not react to light. A test for toxoplasmosis was slightly to moderately elevated, and the patient had chorio-retinitis with visual acuity $2 / 60$. Rucker 
concludes: "To account for disturbed pupillary reflexes and disjunctive nystagmus, the lesion in the brain would be expected to involve the midbrain and pons. Attempting to name the implicating fibre bundles at present leads into the field of speculation".

The case reported by Larsen (1957) is the only one in which a post-mortem examination was performed. The initial signs were horizontal-rotatory nystagmus, changing by way of an irregular phase of alternating rotatory, oblique, and vertical movements, into seesaw nystagmus with a slightly rotatory tendency directed to the left. The signs were most marked on the left. In addition, the patient had mild signs of right pyramidal tract involvement, intention tremor, and signs of increased cerebrospinal pressure. The post-mortem examination revealed a tumour, $5 \times 5 \mathrm{~cm} .(2 \times 2$ in.) at the basis cerebri, reaching from the frontal lobes to the anterior margin of the pons and sending a nodular extension below the left temporal pole. The tumour occupied the entire third ventricle apart from a fine canal superiorly, so there was not a complete fluid block. The brainstem was posteriorly displaced, and the aqueduct of Sylvius was flattened antero-posteriorly. (This is worth noting in connexion with the experimental studies to be mentioned below). The more caudal structures were normal. Microscopic examination showed the tumour to be an oligodendroglioma.

Furtado (1956) published two cases of horizontal disjunctive movements, both of which had come to autopsy. In his former case there was convergent nystagmus with simultaneous flexion in both elbow joints. Microscopic examination revealed disseminated gliomatous nodules in the brainstem, particularly densely arranged in the lamina quadrigemina and adjacent areas. In the latter case, the patient had exhibited oblique downward convergent nystagmus, choked discs, and concentric constriction of the visual fields, as well as slight inhibition of upward ocular movements. Necropsy disclosed a pinealoma exerting marked pressure on the surrounding structures, particularly the superior colliculi, the aqueduct of Sylvius, and the cerebral peduncles. In Furtado's opinion, disjunctive convergent nystagmus is caused by compressive or inflammatory lesions of the peduncles and the lamina quadrigemina. Convergent spasms are known in cases of tumours of the pineal body. In encephalitis involving the peduncles and the region of the cerebral aqueduct, convergent spasms alternating with vertically directed spasms may be seen.

In our Case 1 the signs indicated a vascular, occlusive lesion of the left posterior cerebellar artery, the so-called Wallenberg syndrome, perhaps of the upper part of the left vertebral artery. The latter is suggested by the paresis of the tongue muscles (Walsh, 1957). In addition, there was involvement of the left oculomotor nucleus and the sensory nucleus of the trigeminal nerve and apparently also of the pyramidal tracts, reflected in the mild hyperreflexia of the right lower limb. In the classical Wallenberg syndrome there are more severe signs than in the present case, but the marked 
variations in the course of the vessels, their collaterals, and anastomoses are worth bearing in mind. Moreover, there might be a question of partial occlusion, local oedema, and relative ischaemia, which might also explain the variation in the manifestations. This first patient must have had a mild perhaps only partial occlusion, and he had almost completely recovered within 3 days. The red cells in the spinal fluid indicate that some infarction had taken place. The most remarkable feature of this case was the large area involved by the bulbar manifestations, i.e. from the oculomotor nucleus to the hypoglossal nucleus, but perhaps the signs from the superior nuclei may be ascribed to relative ischaemia. The posterior inferior cerebellar artery is generally stated to supply only the 9 th, 10 th, and 11 th nuclei, while the 12th nucleus is supplied by the fourth part of the vertebral artery. The nuclei situated superior to the 9th nucleus are supplied by the pontine branches of the basilar artery (Cunningham, 1937).

Case $2 \mathrm{had}$, in addition to the seesaw nystagmus, involvement of the oculomotor nerve and perhaps slight involvement of the crossed pyramidal tract, manifesting itself in an extensor plantar response on the right side. On the following day, the signs had already improved. Thus, the condition was somewhat similar to that seen in Case 1, but milder. Unfortunately, further studies could not be performed, as the patient left so quickly.

By electric stimulation of the brainstem of cats, Spiegel and Scala (1937) found that irritation of the grey matter below the posterior commissure or behind this level resulted in upward, conjugate movements of the eyeballs, whereas downward movements could be induced in the posterior part of the optic thalamus or in the medial part of the superior colliculi, i.e. in the vicinity of the pretectal region. Destruction of the grey matter in these areas was followed by loss of up-and-down movements upon cortical stimulation. A small injury of the grey matter in the cranial part of the mesencephalon and the pretectal region might be followed by various partial disturbances of the vertical movements. Keeping seesaw nystagmus in mind, it is interesting that these workers could induce the so-called skew deviation, i.e. one eye looking up while the other looks down.

There is no lack of theories on the vertical movements. Marburg (1939) believed that the left medial longitudinal fasciculus was responsible for upward movements and the right one for downward movements. Muskens (1930) located the upward movements to the medial nuclei of the aqueduct and the downward movements to the lateral nuclei. In the opinion of Godlowsky (1936) the upward movements are controlled by the ventral nucleus reticularis (a thin layer of cells on the lateral aspect of the thalamus) and the downward movements by the nucleus of Darkschewitsch. However, seesaw nystagmus has not been induced experimentally.

Thus, the details of the pathogenesis are still unknown, but seesaw nystagmus appears to occur in the presence of brainstem lesions, be they compressive, inflammatory, or vascular. The experimental findings reviewed 
above indicate that, in the case reported by Larsen (1957), the most important pathogenic factor was the pressure on the region of the aqueduct, secondary to the large basal tumour. Unfortunately, Maddox (1914) gives no indication regarding the aetiology or pathogenesis of the first case on record.

According to the available data, one structure might theoretically be involved in all the cases (apart from that reported by Maddox), viz. the medial longitudinal fasciculus, which is of importance to the co-ordination of ocular movements. This structure extends from the ventral funiculus of the spinal cord to the area surrounding the subthalamus. It consists of association fibres between the nuclei of the ocular muscles as well as of fibres from the vestibular nuclei, the spinal cord, and the sensory nucleus of the trigeminal nerve, and further, includes two tracts with unknown function, including fibres from the nucleus of the posterior commissure, the nucleus of Darkschewitsch ( $c f$. Godlowsky's theory regarding the vertical movements). In this connexion, it is perhaps appropriate to mention the posterior commissure, a large bundle of fibres ventral to the site where the cerebral aqueduct opens into the $3 \mathrm{rd}$ ventricle. This commissure is believed to contain fibres connecting to two superior colliculi, an important zone of transmission between the optic tracts and the nuclei of the ocular muscles. In addition, Marburg (1939) believed that the fibres from the superior vestibular nucleus (of Bechterew) which ascend uncrossed, cross partially in this commissure.

\section{Summary}

Seesaw nystagmus was observed in two cases with short-lived signs of thrombosis in the brainstem arteries. The three previous cases on record are reviewed, and experimental and theoretical considerations are discussed.

It is concluded that the pathogenesis is unknown, but that this extremely rare form of nystagmus appears to occur in the presence of lesions in the brainstem, particularly if there is involvement of the superior parts of the medial longitudinal fasciculus, perhaps in the area of the posterior commissure.

\section{REFERENCES}

Cords, R. (1930). Klin. Mbl. Augenheilk., 3, 630. Cited by Duke-Elder (1949), vol. 4, p. 4233. Cunningham, D. J. (1937). "Text-book of Anatomy", 7th ed., pp. 1184, 1506. Oxford University Press, London.

DUKe-ElDER, S. (1949). "Text-book of Ophthalmology", vol. 4, p. 4232. Kimpton, London. FURTADO, D. (1956). Rev. neurol., 94, 335.

GoDLOWSKY, W. (1936). "Étude expérimentale". Krakow.

LARSEN, V. (1957). Ugeskr. Laeg., 119, 947.

MadDox, E. E. (1914). Proc. roy. Soc. Med., 7, pt. 1. Section for Study of Disease in Children, p. 177.

MARBURG, O. (1939). Laryngoscope, 49, 631.

MUSKenS, L. J. J. (1930). J. comp. Anat., 50, 289.

RUCKER, C. W. (1946). Arch. Ophthal. (Chicago), 35, 301.

SPIEGEL, E. A., and Scala, N. P. (1937). Ibid., 18, 614.

WALSH, F. B. (1957). "Clinical Neuro-Ophthalmology", 2nd ed., pp. 239, 270, 802, 1294. Williams and Wilkins, Baltimore. 